most renal tumors associated with tuberous sclerosis are relatively benign and patients with renal cell carcinoma localized to the kidney have a $75 \%$ chance of recovery following surgery alone.

\title{
DEGEINERATIVE DISORDERS
}

\section{PROGRESSIVE MYOCIONDS EPILEPSY (LAFORA TYPB)}

The diagnosis of Lafora's syndrome, progressive myoclonus epilepsy and intracytoplasmic periodic acid-Schiff-positive inclusions (Lafora bodies), was made by skin biopsy in a 16-year-old girl at the Depts of Pathology and Dermatology, University of Texas Medical Branch, Galveston, TX. She presented because of refractory generalized convulsions. In good health until 8 months previously, she developed progressive incoordination, slurred speech, weakness, and impaired school performance followed after 2 months by her first generalized tonic-clonic seizure and progressive mental deterioration. The family history was negative. Metabolic, endocrine, and infectious disorders were excluded. CT scan was normal. EEG showed "generalized cerebral dysfunction." Round to oval, intracytoplasmic inclusions, strongly PAS-positive, in eccrine duct cells and peripheral nerve bundles of dermis were demonstrated histologically in cryostat- and paraffin-embedded sections and by electronmicroscopy. Skin biopsy, the least invasive method of identifying the Lafora body, was first proposed by Carpenter S. Karpati G (Neurology 1981; 31; 1564). A sumary of other disorders and their characteristic inclusions that may be diagnosed by skin biopsy includes neuronal ceroid lipofuscinoses (Bielschowski and Spielmeyer-Vogt), glycogenosis II (Pompe's), metachromatic leukodystrophy and occasionally, globoid leukodystrophy (Krabbe). (Newton GA et al. Lafora's disease. The role of skin biopsy. Arch Dermatol 1987; 123: 1667-1669).

CONENT. A number of syndromes of progressive myoclonus epilepsy, frequently autosomal recessive in inheritance, have been described. The Lafora type is characterized by a rapidly progressive dementia, myoclonus, and intracytoplasmic Lafora body inclusions demonstrated in neurons, especially localized in the substantia nigra and dentate nucleus, in the heart, liver, muscle, retina, nerves and now in skin. The EEG shows discharges of fast spike-waves and polyspike-waves, photosensitivity, deterioration of background activity, and multifocal abnormalities especially posteriorly. The onset occurs between 6 and 19 years of age and the patient dies within an average of 5.5 years after onset. No enzymatic defect has yet been identified.

The Unverricht-Lundborg types have a slower rate of progression than the Lafora myoclonic epilepsy, with onset at about age 10 years, variable severity of myoclonus, associated cerebellar ataxia, and milder mental symptoms, patients surviving for 15 years and more. This variety known as the Finnish or "Baltic" 
myoclonus epilepsy in which Lafora bodies are absent was exacerbated by phenytoin and benefitted by sodium valproate. (Eldridge R. et al. Lancet 1983; 2: 838).

\section{INIRACRANIAL NBOPLASTS AND ANEURYSES}

\section{EPIILEPSY, TEMPORAL LOBE SCLEROSIS AND TUMORS}

Sixteen of 48 children undergoing temporal lobectomy for temporal lobe epilepsy at the Hospital for Sick Children, Toronto, Ontario, were found to have tumors (12 patients), vascular malformations (3), and arachnoid cyst (1). Nine of these patients had concomitant mesial temporal sclerosis. In 11 of 18 operations (61\%), it was necessary to extend the original cortical excision because of persistent epileptiform activity. The duration of seizures in the 16 patients varied between 1 and 13 years (average $6 \mathrm{yrs}$ ). Memory was impaired in 318 of patients, 398 had behavior problems, and 398 had deteriorating school performance.

Temporal lobectomy was performed on the right side in 8 cases and on the left side in 8 . Four patients had an increased neurological deficit postoperatively and all had contralateral superior quadrantanopic defects. of 15 patients followed for more than 1 year, 9 are seizure free (only 4 on medication), and 7 had more than 50\% reduction in seizure frequency. (Drake $J$ et al. Neurosurgery 1987; 21: 792-797).

COMENT. The authors emphasize that simple excision of the tumor may not eradicate the seizures. The common occurrence of mesial temporal sclerosis in association with mass lesions requires extension of the resection to include removal of the hippocampus as well as the cortex adjacent to the tumor. It is suggested that hippocampal changes are secondary to repetitive seizure activity.

The late detection of cerebral gliomas as a cause of childhood epilepsy has been noted by other investigators. Diagnosis of supratentorial tumors was delayed for an average of 2 years after the initial seizure and 8 of 31 patients continued to have seizures for periods between 3 and 8 years before a tumor was demonstrated in a study of 291 cases at the Mayo $\mathrm{Clinic}$. Seizures occurred in $17 \%$ of the total group - in $25 \%$ of patients with supratentorial tumors and in $12 \%$ of those with infratentorial tumors. They were the initial symptoms in $15 \%$ of patients with supratentorial tumors. Seizures were more common in patients with slowly growing astrocytomas, grades 1 and 2, than in those with more rapidly expanding astrocytomas, grades 3 or 4 . (Pediatrics 1962; 29: 978).

The MRI should permit earlier diagnosis of the temporal lobe glioma as a cause of childhood epilepsy. MRI is superior in defining the extent of gliomas grade 1 and 11 that may be poorly delineated by CT, and MRI should be a complementary examination in 